\title{
Component to compound stimulus transfer
}

\author{
WILLIAM GRINGS, TADAO UNO AND JOSEPHINE FIEBIGER
}

UNIVERSITY OF SOUTHERN CALIFORNIA

\begin{abstract}
Electrodermal responses to pairs of visual stimuli were tested after differential conditioning of the single or component stimuli. On the first test trial, magnitudes tended to be ordered according to principles of response summation. On later trials, extinction variation interacted significantly with transfer variation.

Problem
\end{abstract}

About a decade ago a study was reported demonstrating transfer of conditioned GSR from original learning on single component CSs to tests on pairs of stimuli (Grings \& O'Donnell, 1956). Three classes of colored lights were conditioned differentially through pairing with shock (i.e., consistent reinforcement), consistent nonreinforcement, and nonpresentation during acquisition. Then compounds (pairs of stimuli) were presented. The response to a compound of two reinforced stimuli was found to be significantly greater than the response to any other compound. Results were interpreted to support the assumption that response magnitudes to stimulus aggregates would (under appropriate circumstances) be resultants of the response elicitation capabilities of the component CSs.

Because the principles underlying compound stimulus transfer are basic to many conditioning transfer situations, several related studies were undertaken (e.g., Grings \& Shmelev, 1959; Grings \& Kimmel, 1959)。 The research to be reported here is a direct replication of the earliest (1956) study. Experimental conditions were duplicated carefully, except that new apparatus was used and a somewhat smaller $\mathrm{N}$ was employed. The situation involved training on component stimuli and testing on compounds.

\section{Method}

Twenty-four volunteer Ss were observed first in a GSR conditioning series involving four individual colored light stimuli. Two of the CSs were reinforced $(+)$; one was not reinforced (-); and a fourth, termed a nonpresented cue (0) was introduced only during test series. The stimuli were presented in random blocks of six trials continuing until 10 reinforced trials had been given to two of the stimuli and the other stimulus had been presented 10 times without reinforcement. Test trials on all four single stimuli were interspersed after the fifth and eighth reinforcements in the acquisition series. Following acquisition, two blocks of test trials were given on pairs of the original stimuli. These included pairing of two reinforced cues (++); pairing reinforced and nonreinforced cues (+-); pairing reinforced and nonpresented stimuli $(+0)$; and pairing nonreinforced and nonpresented cues $(-0)$.

The CSs were small colored dots presented on a ground glass screen 30 in in front of S. The UCS was a DC shock delivered from a Grass S5 stimulator to the volar surface of S's forearm. The CS duration was 1 sec.; UCS duration .5 sec.; and CS-UCS interval .5 sec., with intervals controlled by Hunter timers. The GSR was measured as a DC resistance change in a Wheatstone bridge input to an Offner Model 542 recorder.

Further detail on stimulus specification, apparatus, and general procedure can be obtained from the earlier study (Grings \& O'Donnell, 1956).

\section{Results}

The magnitude of response was read in units of the square root of the conductance change occurring in the period from $1 \mathrm{sec}$. to $5 \mathrm{sec}$. after stimulus onset. Since this is a transfer study, it becomes important first to demonstrate differential learning. It was assumed that reinforced experience with a cue would lead to a larger response than would nonreinforced experience. When the results for two test trials in acquisition are combined, the mean GSRs to the reinforced cues are .88 and .87 , the mean response to the nonreinforced cue is .63 , and the mean to the nonpresented cue is .57 . The differences between means for reinforced and nonreinforced cues are significant (ts of 3.10 and 3.35; $\mathrm{df}=23$ ). Those between means for reinforced and nonpresented cues are also significant (ts of 3.22 and 3.25; $\mathrm{df}=23$ ). The difference between response to a nonpresented cue and a nonreinforced cue is not significant $(t=.53)$. The assumption of differential effects of original training is thus supported.

Several ways of evaluating transfer from components to compounds are available. Two extinction testblocks, one of four and one of eight trials, were included. Both blocks included tests of the four combinations $(++,+0,+-$, and -0 ) in varied order from $S$ to $S$. The second test block included tests on the components in addition to tests on compounds. If the amount of extinction over these twelve test trials were not appreciable, it would be possible to combine all data for the transfer test. However, the effects of extinction are extensive and occur very rapidly

The most valid transfer test trials are probably those on the first test trial before the effects of repeated testing on compounds are evident. The mean GSR to the compound first experienced is illustrated in Fig. 1. It will be noted that the relative magnitudes are as 


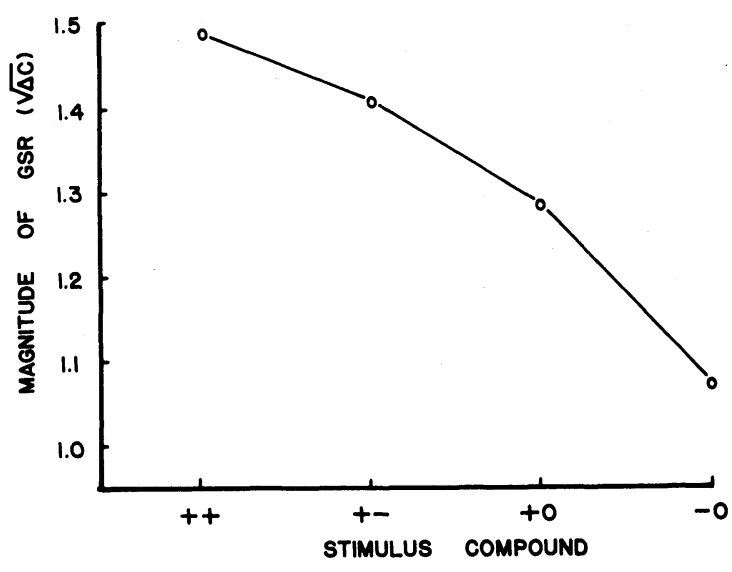

Fig. 1. Mean GSR to compounds on first test trial.

predicted. This comparison, however, is based on independent samples of six Ss each, with the result that the trend does not achieve statistical significance.

The extent to which extinction is a factor can be seen by an analysis of variance for the first four extinction trials (first test block) which included one presentation of each test compound. These results indicate a significant extinction effect $(F=12.12 ; \mathrm{df}=3,57)$ and a significant interaction between compounds and extinction $(\mathrm{F}=3.76 ; \mathrm{df}=9,57)$. The curve in Fig. 1 represents the first test trial. By the fourth test trial the combination of two reinforced components had shown much greater extinction than had the response to either compound involving nonreinforced and nonpresented components. (See Fig. 2.) Rapid extinction also complicated comparisons of response to components and compounds together during the second extinction (test) block.

\section{Diseussion}

Two major conclusions are drawn from the present study. One is that initial transfer from components to compounds appears to follow closely principles of simple response summation. But with more than one test trial it is found that extinction effects interact with simple transfer causing response magnitudes to vary from what would be expected from simple response summation.

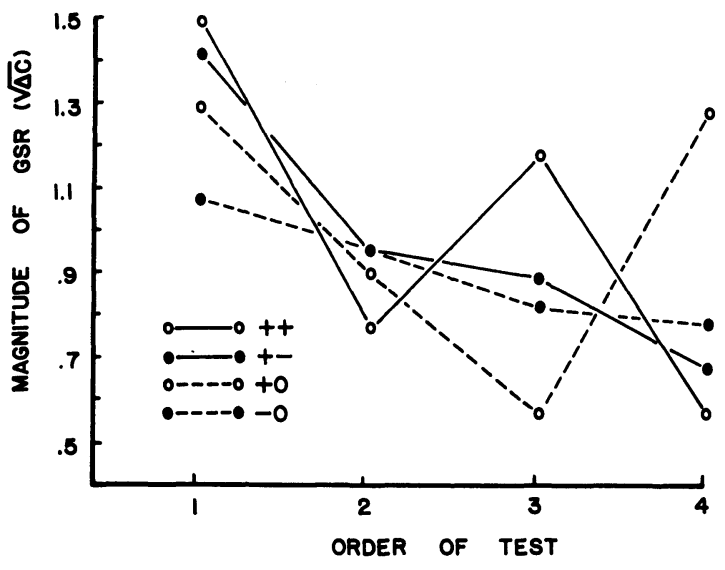

Fig. 2. Mean GSR to compounds on four test trials.

If compound stimulus summation independent of extinction is to be demonstrated more adequately, between groups design with larger Ns is indicated. Such a design would permit evaluating transfer on a single first test trial. Other means for evaluating such transfer may involve the periodic interspersing of transfer tests throughout more prolonged acquisition series. A second related problem needing further study centers a round the principles governing the interaction of compound stimulus transfer and extinction. Relevant in the present context is the fact that continuous (with two interspersed test trials) rather than partial reinforcement was used in acquisition training of reinforced stimuli. Extinction might have been less rapid with a partial reinforcement paradigm.

\section{Referenees}

Grings, W., \& Kimmel, H. Compound stimulus transfer for different sense modalities. Psychol. Reps., 1959, 5, 253-260.

Grings, W., \& O'Donnell, D. Magnitude of response to compounds of discriminated stimuli. J. exp. Psychol., 1956, 52, 354-358.

Grings, W., \& Shmelev, V. Changes in GSR to a single stimulus as a result of training on a compound stimulus. J. exp. Psychol., 1959, 58, 129-133.

\section{Note}

1. This research was supported in part by Grant MH 3916 from the National Institute of Mental Health. 